PUPT-1616

Imperial/TP/95-96/41

hep-th/9604166

\title{
Intersecting M-branes as Four-Dimensional Black Holes
}

\author{
I.R. Klebanov* \\ Joseph Henry Laboratories \\ Princeton University, Princeton, NJ 08544 \\ and \\ A.A. Tseytlin ${ }^{\star \dagger}$ \\ Theoretical Physics Group, Blackett Laboratory \\ Imperial College, London SW' 2BZ, U.K.
}

\begin{abstract}
We present two $1 / 8$ supersymmetric intersecting p-brane solutions of 11-dimensional supergravity which upon compactification to four dimensions reduce to extremal dyonic black holes with finite area of horizon. The first solution is a configuration of three intersecting 5 -branes with an extra momentum flow along the common string. The second describes a system of two 2-branes and two 5-branes. Related (by compactification and T-duality) solution of type IIB theory corresponds to a completely symmetric configuration of four intersecting 3-branes. We suggest methods for counting the BPS degeneracy of three intersecting 5-branes which, in the macroscopic limit, reproduce the Bekenstein-Hawking entropy.
\end{abstract}

April 1996

* e-mail address: klebanov@puhep1.princeton.edu

* e-mail address: tseytlin@ic.ac.uk

$\dagger$ On leave from Lebedev Physics Institute, Moscow. 


\section{Introduction}

The existence of supersymmetric extremal dyonic black holes with finite area of the horizon provides a possibility of a statistical understanding [1] of the Bekenstein-Hawking entropy from the point of view of string theory [2, 3, [1]. Such black hole solutions are found in four [5,6, [7] and five [4,8] dimensions but not in $D>5$ [9, 10]. While the D-brane BPS state counting derivation of the entropy is relatively straightforward for the $D=5$ black holes [4]:11], it is less transparent in the $D=4$ case, a complication being the presence of a solitonic 5-brane or Kaluza-Klein monopole in addition to a D-brane configuration in the descriptions used in [12,13].

One may hope to find a different lifting of the dyonic $D=4$ black hole to $D=10$ string theory that may correspond to a purely D-brane configuration. A related question is about the embedding of the $D=4$ dyonic black holes into $D=11$ supergravity (M-theory) which would allow to reproduce their entropy by counting the corresponding BPS states using the M-brane approach similar to the one applied in the $D=5$ black hole case in [14.

As was found in 15], the (three-charge, finite area) $D=5$ extremal black hole can be represented in M-theory by a configuration of orthogonally intersecting 2-brane and 5 -brane (i.e. $2 \perp 5$ ) with a momentum flow along the common string, or by configuration of three 2-branes intersecting over a point $(2 \perp 2 \perp 2)$. A particular embedding of (four-charge, finite area) $D=4$ black hole into $D=11$ theory given in 15 can be interpreted as a similar $2 \perp 5$ configuration 'superposed' with a Kaluza-Klein monopole.

Below we shall demonstrate that it is possible to get rid of the complication associated with having the Kaluza-Klein monopole. There exists a simple 1/8 supersymmetric configuration of four intersecting M-branes $(2 \perp 2 \perp 5 \perp 5)$ with diagonal $D=11$ metric. Upon compactification along six isometric directions it reduces to the dyonic $D=4$ black hole with finite area and all scalars being regular at the horizon.

The corresponding $2 \perp 2 \perp 4 \perp 4$ solution of type IIA $D=10$ superstring theory (obtained by dimensional reduction along a direction common to the two 5 -branes) is $T$-dual to a $D=10$ solution of type IIB theory which describes a remarkably symmetric configuration of four intersecting 3-branes. 1

1 Similar D-brane configuration was discussed in [16, 17]. Note that it is a combination of four and not three intersecting 3-branes that is related (for the special choice of equal charges) to the non-dilatonic $(a=0)$ RN $D=4$ black hole. $T$-dual configuration of one 0-brane and three intersecting 4-branes of type IIA theory was considered in [18]. 
Our discussion will follow closely that of [15] where an approach to constructing intersecting supersymmetric p-brane solutions (generalising that of [19]) was presented.2 The supersymmetric configurations of two or three intersecting 2- and 5-branes of $D=11$ supergravity which preserve $1 / 4$ or $1 / 8$ of maximal supersymmetry are $2 \perp 2,5 \perp 5,2 \perp 5$, $2 \perp 2 \perp 2,5 \perp 5 \perp 5,2 \perp 2 \perp 5$ and $2 \perp 5 \perp 5$. Two 2-branes can intersect over a point, two 5 branes - over a 3-brane (which in turn can intersect over a string), 2-brane and 5-brane can intersect over a string [19]. There exists a simple 'harmonic function' rule which governs the construction of composite supersymmetric p-brane solutions in both $D=10$ and $D=11$ : a separate harmonic function is assigned to each constituent $1 / 2$ supersymmetric p-brane.

Most of the configurations with four intersecting M-branes, namely, $2 \perp 2 \perp 2 \perp 2$, $2 \perp 2 \perp 2 \perp 5$ and $5 \perp 5 \perp 5 \perp 2$ are $1 / 16$ supersymmetric and have transverse $x$-space dimension equal to two $(5 \perp 5 \perp 5 \perp 5$ configuration with 5 -branes intersecting over 3 -branes to preserve supersymmetry does not fit into 11-dimensional space-time). Being described in terms of harmonic functions of $x$ they are thus not asymptotically flat in transverse directions. There exists, however, a remarkable exception - the configuration $2 \perp 2 \perp 5 \perp 5$ which (like $5 \perp 2 \perp 2,5 \perp 5 \perp 2$ and $5 \perp 5 \perp 5$ ) has transverse dimension equal to three and the fraction of unbroken supersymmetry equal to $1 / 8$ (Section 3 ). Upon compactification to $D=4$ it reduces to the extremal dyonic black hole with four different charges and finite area of the horizon.

Similar $D=4$ black hole background can be obtained also from the the 'boosted' version of the $D=115 \perp 5 \perp 5$ solution [15] (Section 2) l $^{1}$ as well from the $3 \perp 3 \perp 3 \perp 3$ solution of $D=10$ type IIB theory (Section 4 ). The two $D=11$ configurations $5 \perp 5 \perp 5+$ 'boost' and $2 \perp 2 \perp 5 \perp 5$ reduce in $D=10$ to $0 \perp 4 \perp 4 \perp 4$ and $2 \perp 2 \perp 4 \perp 4$ solutions of $D=10$ type IIA theory which are related by $T$-duality.

In Section 5 we shall suggest methods for counting the BPS entropy of three intersecting 5-branes which reproduce the Bekenstein-Hawking entropy of the $D=4$ black hole. This seems to explain the microscopic origin of the entropy directly in 11-dimensional terms.

2 Intersecting p-brane solutions in 19, 15 and below are isometric in all directions internal to all constituent p-branes (the background fields depend only on the remaining common transverse directions). They are different from possible virtual configurations where, e.g., a (p-2)-brane ends (in transverse radial direction) on a p-brane [20]. A configuration of p-brane and $\mathrm{p}^{\prime}$-brane intersecting in $\mathrm{p}+\mathrm{p}^{\prime}$-space may be also considered as a special anisotropic $\mathrm{p}+\mathrm{p}^{\prime}$-brane. There may exist more general solutions (with constituent p-branes effectively having different transverse spaces [19,21]) which may 'interpolate' between intersecting p-brane solutions and solutions with one p-brane ending on another in the transverse direction of the latter.

3 The 'boost' along the common string corresponds to a Kaluza-Klein electric charge part in the $D=11$ metric which is 'dual' to a Kaluza-Klein monopole part present in the $D=11$ embedding of dyonic black hole in [15]. 


\section{2. 'Boosted' $5 \perp 5 \perp 5$ solution of $D=11$ theory}

The $D=11$ background corresponding to $5 \perp 5 \perp 5$ configuration [19] is [15]

$$
\begin{gathered}
d s_{11}^{2}=\left(F_{1} F_{2} F_{3}\right)^{-2 / 3}\left[F_{1} F_{2} F_{3}\left(-d t^{2}+d y_{1}^{2}\right)\right. \\
\left.+F_{2} F_{3}\left(d y_{2}^{2}+d y_{3}^{2}\right)+F_{1} F_{3}\left(d y_{4}^{2}+d y_{5}^{2}\right)+F_{1} F_{2}\left(d y_{6}^{2}+d y_{7}^{2}\right)+d x_{s} d x_{s}\right] \\
\mathcal{F}_{4}=3\left(* d F_{1}^{-1} \wedge d y_{2} \wedge d y_{3}+* d F_{2}^{-1} \wedge d y_{4} \wedge d y_{5}+* d F_{3}^{-1} \wedge d y_{6} \wedge d y_{7}\right) .
\end{gathered}
$$

Here $\mathcal{F}_{4}$ is the 4 -form field strength and $F_{i}$ are the inverse powers of harmonic functions of $x_{s}(s=1,2,3)$. In the simplest 1 -center case discussed below $F_{i}^{-1}=1+P_{i} / r \quad\left(r^{2}=x_{s} x_{s}\right)$. The $*$-duality is defined with respect to the transverse 3 -space. The coordinates $y_{n}$ internal to the three 5 -branes can be identified according to the $F_{i}$ factors inside the square brackets in the metric: $\left(y_{1}, y_{4}, y_{5}, y_{6}, y_{7}\right)$ belong to the first 5-brane, $\left(y_{1}, y_{2}, y_{3}, y_{6}, y_{7}\right)$ to the second and $\left(y_{1}, y_{2}, y_{3}, y_{4}, y_{5}\right)$ to the third. 5-branes intersect over three 3 -branes which in turn intersect over a common string along $y_{1}$. If $F_{2}=F_{3}=1$ the above background reduces to the single 5-brane solution [22] with the harmonic function $H=F_{1}^{-1}$ independent of the two of transverse coordinates (here $y_{2}, y_{3}$ ). The case of $F_{3}=1$ describes two 5-branes intersecting over a 3-brane 1 The special case of $F_{1}=F_{2}=F_{3}$ is the solution found in [19].

Compactifying $y_{1}, . ., y_{7}$ on circles we learn that the effective 'radii' (scalar moduli fields in $D=4$ ) behave regularly both at $r=\infty$ and at $r=0$ with the exception of the 'radius' of $y_{1}$. It is possible to stabilize the corresponding scalar by adding a 'boost' along the common string. The metric of the resulting more general solution [15] is (the expression for $\mathcal{F}_{4}$ remains the same)

$$
\begin{gathered}
d s_{11}^{2}=\left(F_{1} F_{2} F_{3}\right)^{-2 / 3}\left[F_{1} F_{2} F_{3}\left(d u d v+K d u^{2}\right)\right. \\
\left.+F_{2} F_{3}\left(d y_{2}^{2}+d y_{3}^{2}\right)+F_{1} F_{3}\left(d y_{4}^{2}+d y_{5}^{2}\right)+F_{1} F_{2}\left(d y_{6}^{2}+d y_{7}^{2}\right)+d x_{s} d x_{s}\right] .
\end{gathered}
$$

Here $u=y_{1}-t, v=2 t$ and $K$ is a harmonic function of the three coordinates $x_{s}$. A non-trivial $K=1+Q / r$ describes a momentum flow along the string direction. 0

4 The corresponding $1 / 4$ supersymmetric background also has 3 -dimensional transverse space and reduces to a $D=4$ black hole with two charges (it has $a=1$ black hole metric when two charges are equal). The $5 \perp 5$ configuration compactified to $D=10$ gives $4 \perp 4$ solution of type IIA theory which is $T$-dual to $3 \perp 3$ solution of type IIB theory.

5 The metric (2.3) with $F_{i}=1$ (i.e. $d s^{2}=-K^{-1} d t^{2}+K\left[d y_{1}+\left(K^{-1}-1\right) d t\right]^{2}+d y_{n} d y_{n}+d x_{s} d x_{s}$ ) reduces upon compactification along $y_{1}$ direction to the $D=10$ type IIA R-R 0-brane background [23] with $Q$ playing the role of the KK electric charge. 
$Q$ also has an interpretation of The $D=11$ metric (2.3) is regular at the $r=0$ horizon and has a non-zero 9 -area of the horizon (we assume that all $y_{n}$ have period $L$ )

$$
A_{9}=4 \pi L^{7}\left[r^{2} K^{1 / 2}\left(F_{1} F_{2} F_{3}\right)^{-1 / 2}\right]_{r \rightarrow 0}=4 \pi L^{7} \sqrt{Q P_{1} P_{2} P_{3}} .
$$

Compactification along $y_{2}, \ldots y_{7}$ leads to a solitonic $D=5$ string. Remarkably, the corresponding 6 -volume is constant so that one gets directly the Einstein-frame metric

$$
d s_{5}^{2}=H^{-1}\left(d u d v+K d u^{2}\right)+H^{2} d x_{s} d x_{s}, \quad H \equiv\left(F_{1} F_{2} F_{3}\right)^{-1 / 3} .
$$

Further compactification along $y_{1}$ or $u$ gives the $D=4$ (Einstein-frame) metric which is isomorphic to the one of the dyonic black hole [6]

$$
\begin{gathered}
d s_{4}^{2}=-\lambda(r) d t^{2}+\lambda^{-1}(r)\left(d r^{2}+r^{2} d \Omega_{2}^{2}\right), \\
\lambda(r)=\sqrt{K^{-1} F_{1} F_{2} F_{3}}=\frac{r^{2}}{\sqrt{(r+Q)\left(r+P_{1}\right)\left(r+P_{2}\right)\left(r+P_{3}\right)}} .
\end{gathered}
$$

Note, however, that in contrast to the dyonic black hole background of [6] which has two electric and two magnetic charges here there is one electric (Kaluza-Klein) and 3 magnetic charges. From the $D=4$ point of view the two backgrounds are related by $U$-duality. The corresponding 2-area of the $r=0$ horizon is of course $A_{9} / L^{7}$.

In the special case when all 4 harmonic functions are equal $\left(K=F_{i}=H^{-1}\right)$ the metric (2.3) becomes

$$
\begin{gathered}
d s_{11}^{2}=H^{-1} d u d v+d u^{2}+d y_{2}^{2}+\ldots+d y_{6}^{2}+H^{2} d x_{s} d x_{s} \\
=-H^{-2} d t^{2}+H^{2} d x_{s} d x_{s}+\left[d y_{1}+\left(H^{-1}-1\right) d t\right]^{2}+d y_{2}^{2}+\ldots+d y_{6}^{2},
\end{gathered}
$$

and corresponds to a charged solitonic string in $D=5$ or the Reissner-Nordström $(a=0)$ black hole in $D=4$ ('unboosted' $5 \perp 5 \perp 5$ solution with $K=1$ and equal $F_{i}$ reduces to $a=1 / \sqrt{3}$ dilatonic $D=4$ black hole [19]).

A compactification of this $5 \perp 5 \perp 5+$ 'boost' configuration to $D=10$ along $y_{1}$ gives a type IIA solution corresponding to three 4-branes intersecting over 2-branes plus additional Kaluza-Klein (Ramond-Ramond vector) electric charge background, or, equivalently, to the $0 \perp 4 \perp 4 \perp 4$ configuration of three 4 -branes intersecting over 2-branes which in turn intersect over a 0-brane. If instead we compactify along a direction common only to two of the three 5-branes we get $4 \perp 4 \perp 5+$ 'boost' type IIA solution. ${ }^{3}$ Other related solutions of type IIA and IIB theories can be obtained by applying $T$-duality and $S L(2, Z)$ duality.

6 This may be compared to another type IIA configuration (consisting of solitonic 5-brane lying within a R-R 6-brane, both being intersected over a 'boosted' string by a R-R 2-brane) which also reduces [12,15] to the dyonic $D=4$ black hole. 


\section{3. $2 \perp 2 \perp 5 \perp 5$ solution of $D=11$ theory}

Solutions with four intersecting M-branes are constructed according to the rules discussed in [15]. The $2 \perp 2 \perp 5 \perp 5$ configuration is described by the following background

$$
\begin{gathered}
d s_{11}^{2}=\left(T_{1} T_{2}\right)^{-1 / 3}\left(F_{1} F_{2}\right)^{-2 / 3}\left[-T_{1} T_{2} F_{1} F_{2} d t^{2}\right. \\
\left.+T_{1} F_{1} d y_{1}^{2}+T_{1} F_{2} d y_{2}^{2}+T_{2} F_{1} d y_{3}^{2}+T_{2} F_{2} d y_{4}^{2}+F_{1} F_{2}\left(d y_{5}^{2}+d y_{6}^{2}+d y_{7}^{2}\right)+d x_{s} d x_{s}\right] \\
\mathcal{F}_{4}=-3 d t \wedge\left(d T_{1} \wedge d y_{1} \wedge d y_{2}+d T_{2} \wedge d y_{3} \wedge d y_{4}\right) \\
+3\left(* d F_{1}^{-1} \wedge d y_{2} \wedge d y_{4}+* d F_{2}^{-1} \wedge d y_{1} \wedge d y_{3}\right) .
\end{gathered}
$$

Here $T_{i}^{-1}$ are harmonic functions corresponding to the 2-branes and $F_{i}^{-1}$ are harmonic functions corresponding to the 5 -branes, i.e.

$$
T_{i}^{-1}=1+\frac{Q_{i}}{r}, \quad F_{i}^{-1}=1+\frac{P_{i}}{r} .
$$

$\left(y_{1}, y_{2}\right)$ belong to the first and $\left(y_{3}, y_{4}\right)$ to the second 2-brane. $\left(y_{1}, y_{3}, y_{5}, y_{6}, y_{7}\right)$ and $\left(y_{2}, y_{4}, y_{5}, y_{6}, y_{7}\right)$ are the coordinates of the two 5-branes. Each 2-brane intersects each 5 -brane over a string. 2-branes intersect over a 0 -brane $(x=0)$ and 5 -branes intersect over a 3-brane.

Various special cases include, in particular, the 2-brane solution [24] $\left(T_{2}=F_{1}=F_{2}=\right.$ $1)$, as well as $5 \perp 5\left(T_{1}=T_{2}=1\right)$ [19] and $2 \perp 5\left(T_{1}=F_{2}=1\right), 2 \perp 2 \perp 5\left(F_{2}=1\right), 2 \perp 5 \perp 5$ $\left(T_{2}=1\right)$ 15 configurations (more precisely, their limits when the harmonic functions do not depend on a number of transverse coordinates).

As in the case of the $5 \perp 5 \perp 5+$ 'boost' solution (2.3), 2.2), the metric (3.1) is regular at the $r=0$ horizon (in particular, all internal $y_{n}$-components smoothly interpolate between finite values at $r \rightarrow \infty$ and $r \rightarrow 0$ ) with the 9-area of the horizon being (cf.(2.4))

$$
A_{9}=4 \pi L^{7}\left[r^{2}\left(T_{1} T_{2} F_{1} F_{2}\right)^{-1 / 2}\right]_{r \rightarrow 0}=4 \pi L^{7} \sqrt{Q_{1} Q_{2} P_{1} P_{2}} .
$$

The compactification of $y_{n}$ on 7 -torus leads to a $D=4$ background with the metric which is again the dyonic black hole one (2.6), now with

$$
\lambda(r)=\sqrt{T_{1} T_{2} F_{1} F_{2}}=\frac{r^{2}}{\sqrt{\left(r+Q_{1}\right)\left(r+Q_{2}\right)\left(r+P_{1}\right)\left(r+P_{2}\right)}} .
$$

In addition, there are two electric and two magnetic vector fields (as in [6]) and also 7 scalar fields. The two electric and two magnetic charges are directly related to the 2-brane and 5-brane charges (cf. (3.2)).

When all 4 harmonic functions are equal $\left(T_{i}^{-1}=F_{i}^{-1}=H\right)$ the metric (3.1) becomes (cf. $(2.8)$ )

$$
d s_{11}^{2}=-H^{-2} d t^{2}+H^{2} d x_{s} d x_{s}+d y_{1}^{2}+\ldots+d y_{7}^{2},
$$

i.e. describes a direct product of a $D=4$ Reissner-Nordström black hole and a 7-torus.

Thus there exists an embedding of the dyonic $D=4$ black holes into $D=11$ theory which corresponds to a remarkably symmetric combination of M-branes only. In contrast to the embeddings with a Kaluza-Klein monopole [15] or electric charge ('boost') (2.3), (2.8) it has a diagonal $D=11$ metric. 


\section{4. $3 \perp 3 \perp 3 \perp 3$ solution of type IIB theory}

Dimensional reduction of the background (3.1), (3.2) to $D=10$ along a direction common to the two 5-brane (e.g. $y_{7}$ ) gives a type IIA theory solution representing the R-R p-brane configuration $2 \perp 2 \perp 4 \perp 4$. This configuration is $T$-dual to $0 \perp 4 \perp 4 \perp 4$ one which is the dimensional reduction of the $5 \perp 5 \perp 5+$ 'boost' solution. This suggests also a relation between the two $D=11$ configurations discussed in Sections 2 and 3 .

$T$-duality along one of the two directions common to 4 -branes transforms $2 \perp 2 \perp 4 \perp 4$ into the $3 \perp 3 \perp 3 \perp 3$ solution of type IIB theory. The explicit form of the latter can be found also directly in $D=10$ type IIB theory (i.e. independently of the above $D=11$ construction) using the method of [15], where the $1 / 4$ supersymmetric solution corresponding to two intersecting 3 -branes was given. One finds the following $D=10$ metric and self-dual 5 -form (other $D=10$ fields are trivial)

$$
\begin{gathered}
d s_{10}^{2}=\left(T_{1} T_{2} T_{3} T_{4}\right)^{-1 / 2}\left[-T_{1} T_{2} T_{3} T_{4} d t^{2}\right. \\
\left.+T_{1} T_{2} d y_{1}^{2}+T_{1} T_{3} d y_{2}^{2}+T_{1} T_{4} d y_{3}^{2}+T_{2} T_{3} d y_{4}^{2}+T_{2} T_{4} d y_{5}^{2}+T_{3} T_{4} d y_{6}^{2}+d x_{s} d x_{s}\right] \\
\mathcal{F}_{5}=d t \wedge\left(d T_{1} \wedge d y_{1} \wedge d y_{2} \wedge d y_{3}+d T_{2} \wedge d y_{1} \wedge d y_{4} \wedge d y_{5}\right. \\
\left.+d T_{3} \wedge d y_{2} \wedge d y_{4} \wedge d y_{6}+d T_{4} \wedge d y_{3} \wedge d y_{5} \wedge d y_{6}\right) \\
+* d T_{1}^{-1} \wedge d y_{4} \wedge d y_{5} \wedge d y_{6}+* d T_{2}^{-1} \wedge d y_{2} \wedge d y_{3} \wedge d y_{6} \\
+* d T_{3}^{-1} \wedge d y_{1} \wedge d y_{3} \wedge d y_{5}+* d T_{4}^{-1} \wedge d y_{1} \wedge d y_{2} \wedge d y_{4}
\end{gathered}
$$

The coordinates of the four 3 -branes are $\left(y_{1}, y_{2}, y_{3}\right),\left(y_{1}, y_{4}, y_{5}\right),\left(y_{2}, y_{4}, y_{6}\right)$ and $\left(y_{3}, y_{5}, y_{6}\right)$, i.e. each pair of 3-branes intersect over a string and all 6 strings intersect at one point. $T_{i}$ are the inverse harmonic functions corresponding to each 3-brane, $T_{i}^{-1}=1+Q_{i} / r$. Like the $2 \perp 2 \perp 5 \perp 5$ background of $D=11$ theory this $D=10$ solution is $1 / 8$ supersymmetric, has 3 -dimensional transverse space and diagonal $D=10$ metric.

Its special cases include the single 3-brane [23,25] with harmonic function independent of 3 of 6 transverse coordinates $\left(T_{2}=T_{3}=T_{4}=1\right), 3 \perp 3$ solution found in 15 , $\left(T_{3}=T_{4}=\right.$ 1 ) and also $3 \perp 3 \perp 3$ configuration $\left(T_{4}=1\right)$. The $1 / 8$ supersymmetric $3 \perp 3 \perp 3$ configuration also has 3-dimensional transverse spacel but the corresponding $D=10$ metric

$$
\begin{gathered}
d s_{10}^{2}=\left(T_{1} T_{2} T_{3}\right)^{-1 / 2}\left[-T_{1} T_{2} T_{3} d t^{2}\right. \\
\left.+T_{1} T_{2} d y_{1}^{2}+T_{1} T_{3} d y_{2}^{2}+T_{1} d y_{3}^{2}+T_{2} T_{3} d y_{4}^{2}+T_{2} d y_{5}^{2}+T_{3} d y_{6}^{2}+d x_{s} d x_{s}\right]
\end{gathered}
$$

7 Similar configurations of three and four intersecting 3-branes, and, in particular, their invariance under the $1 / 8$ fraction of maximal supersymmetry were discussed in $D$-brane representation in 17, 16. 
is singular at $r=0$ and has zero area of the $r=0$ horizon. 8

As in the two $D=11$ cases discussed in the previous sections, the metric of the $3 \perp 3 \perp 3 \perp 3$ solution (4.1) has $r=0$ as a regular horizon with finite 8-area (cf.(2.4),(3.4))

$$
A_{8}=4 \pi L^{6}\left[r^{2}\left(T_{1} T_{2} T_{1} T_{2}\right)^{-1 / 2}\right]_{r \rightarrow 0}=4 \pi L^{6} \sqrt{Q_{1} Q_{2} Q_{3} Q_{4}} .
$$

$A_{8} / L^{6}$ is the area of the horizon of the corresponding dyonic $D=4$ black hole with the metric (2.6) and

$$
\lambda(r)=\sqrt{T_{1} T_{2} T_{3} T_{4}}=\frac{r^{2}}{\sqrt{\left(r+Q_{1}\right)\left(r+Q_{2}\right)\left(r+Q_{3}\right)\left(r+Q_{4}\right)}} .
$$

The gauge field configuration here involves 4 pairs of equal electric and magnetic charges. When all charges are equal, the $3 \perp 3 \perp 3 \perp 3$ metric (4.1) compactified to $D=4$ reduces to the $a=0$ black hole metric (while the $3 \perp 3 \perp 3$ metric (4.3) reduces to the $a=1 / \sqrt{3}$ black hole metric [26]).

\section{Entropy of $D=4$ Reissner-Nordström black hole}

Above we have demonstrated the existence of supersymmetric extremal $D=11$ and $D=10$ configurations with finite entropy which are built solely out of the fundamental $p$-branes of the corresponding theories (the 2-branes and the 5-branes of the M-theory and the 3 -branes of type IIB theory) and reduce upon compactification to $D=4$ dyonic black hole backgrounds with regular horizon.

Namely, there exists an emdedding of a four dimensional dyonic black hole (in particular, of the non-dilatonic Reissner-Nordström black hole) into $D=11$ theory which corresponds to a combination of M-branes only. This may allow an application of the approach similar to the one of [14] to the derivation of the entropy (3.4) by counting the number of different BPS excitations of the $2 \perp 2 \perp 5 \perp 5$ M-brane configuration.

The $3 \perp 3 \perp 3 \perp 3$ configuration represents an embedding of the $1 / 8$ supersymmetric dyonic $D=4$ black hole into type IIB superstring theory which is remarkable in that all four charges enter symmetrically. It is natural to expect that there should exist a microscopic counting of the BPS states which reproduces the Bekenstein-Hawking entropy in a ( $U$-duality invariant) way that treats all four charges on an equal footing.

Although we hope to eventually attain a general understanding of this problem, in what follows we shall discuss the counting of BPS states for one specific example discussed above: the M-theory configuration (2.3),(2.2) of the three intersecting 5-branes with a common line. Even though the counting rules of M-theory are not entirely clear, we see an advantage to doing this from M-theory point of view as compared to previous discussions in the context of string theory [12,13]: the 11-dimensional problem is more symmetric. Furthermore, apart from the entropy problem, we may learn something about the Mtheory.

8 This is similar to what one finds for the 'unboosted' $5 \perp 5 \perp 5$ configuration (2.1),(2.2). As is well-known from 4-dimensional point of view, one does need four charges to get a regular behaviour of scalars near the horizon and finite area. 


\subsection{Charge quantization in M-theory and the Bekenstein-Hawking entropy}

Upon dimensional reduction to four dimensions, the boosted $5 \perp 5 \perp 5$ solution (2.3), (2.2), reduces to the 4-dimensional black hole with three magnetic charges, $P_{1}, P_{2}$ and $P_{3}$, and an electric charge $Q$. The electric charge is proportional to the momentum along the intersection string of length $L, \mathcal{P}=2 \pi N / L$. The general relation between the coefficient $Q$ in the harmonic function $K$ appearing in (2.3) and the momentum along the $D=5$ string (cf.(2.5)) wound around a compact dimension of length $L$ is (see e.g. [27)

$$
Q=\frac{2 \kappa_{D-1}^{2}}{(D-4) \omega_{D-3}} \cdot \frac{2 \pi N}{L}=\frac{\kappa_{4}^{2} N}{L}=\frac{\kappa^{2} N}{L^{8}},
$$

where $\kappa_{4}^{2} / 8 \pi$ and $\kappa^{2} / 8 \pi$ are the Newton's constants in 4 and 11 dimensions. All toroidal directions are assumed to have length $L$.

The three magnetic charges are proportional to the numbers $n_{1}, n_{2}, n_{3}$ of 5 -branes in the (14567), (12367), and the (12345) planes, respectively (see (2.1),(2.3)). The complete symmetry between $n_{1}, n_{2}$ and $n_{3}$ is thus automatic in the 11-dimensional approach. The precise relation between $P_{i}$ and $n_{i}$ is found as follows. The charge $q_{5}$ of a $D=115$-brane which is spherically symmetric in transverse $d+2 \leq 5$ dimensions is proportional to the coefficient $P$ in the corresponding harmonic function. For $d+2=3$ appropriate to the present case (two of five transverse directions are isotropic, or, equivalently, there is a periodic array of 5-branes in these compact directions) we get

$$
q_{5}=\frac{\omega_{d+1} d}{\sqrt{2} \kappa} P \rightarrow \frac{\omega_{2} L^{2}}{\sqrt{2} \kappa} P=\frac{4 \pi L^{2}}{\sqrt{2} \kappa} P .
$$

At this point we need to know precisely how the 5-brane charge is quantized. This was discussed in [9], but we repeat the argument here for completeness. A different argument leading to equivalent results was presented earlier in [28]. Upon compactification on a circle of length $L$, the M-theory reduces to type IIA string theory where all charge quantization rules are known. We use the fact that double dimensional reduction turns a 2-brane into a fundamental string, and a 5-brane into a Dirichlet 4-brane. Hence, we have

$$
T_{2} \kappa^{2}=T_{1} \kappa_{10}^{2}, \quad T_{5} \kappa^{2}=T_{4} \kappa_{10}^{2},
$$

where the 10-dimensional gravitational constant is expressed in terms of the 11-dimensional one by $\kappa_{10}^{2}=\kappa^{2} / L$. The charge densities are related to the tensions by

$$
q_{2}=\sqrt{2} \kappa T_{2}, \quad q_{5}=\sqrt{2} \kappa T_{5}
$$

and we assume that the minimal Dirac condition is satisfied, $q_{2} q_{5}=2 \pi$. These relations, together with the 10-dimensional expressions [29]

$$
\kappa_{10}=g\left(\alpha^{\prime}\right)^{2}, \quad T_{1}=\frac{1}{2 \pi \alpha^{\prime}}, \quad \kappa_{10} T_{4}=\frac{1}{2 \sqrt{\pi \alpha^{\prime}}},
$$


fix all the M-theory quantities in terms of $\alpha^{\prime}$ and the string coupling constant, $g$. In particular, we find

$$
\kappa^{2}=\frac{g^{3}\left(\alpha^{\prime}\right)^{9 / 2}}{4 \pi^{5 / 2}}, \quad L=\frac{g \sqrt{\alpha^{\prime}}}{4 \pi^{5 / 2}} .
$$

The tensions turn out to be

$$
T_{2}=\frac{2 \pi^{3 / 2}}{g\left(\alpha^{\prime}\right)^{3 / 2}}, \quad T_{5}=\frac{2 \pi^{2}}{g^{2}\left(\alpha^{\prime}\right)^{3}} .
$$

Note that $T_{2}$ is identical to the tension of the Dirichlet 2-brane of type IIA theory, while $T_{5}$ - to the tension of the solitonic 5 -brane. This provides a nice check on our results, since single dimensional reduction indeed turns the M-theory 2-brane into the Dirichlet 2-brane, and the M-theory 5-brane into the solitonic 5-brane. Note that the M-brane tensions satisfy the relation $2 \pi T_{5}=T_{2}^{2}$, which was first derived in [28 using toroidal compactification to type IIB theory in 9 dimensions. This serves as yet another consistency check.

It is convenient to express our results in pure M-theory terms. The charges are quantized according to 9

$$
\begin{gathered}
q_{2}=\sqrt{2} \kappa T_{2}=n \sqrt{2}\left(2 \kappa \pi^{2}\right)^{1 / 3} \\
q_{5}=\sqrt{2} \kappa T_{5}=n \sqrt{2}\left(\frac{\pi}{2 \kappa}\right)^{1 / 3},
\end{gathered}
$$

i.e.

$$
P_{i}=\frac{n_{i}}{2 \pi L^{2}}\left(\frac{\pi \kappa^{2}}{2}\right)^{1 / 3}
$$

The resulting expression for the Bekenstein-Hawking entropy of the extremal ReissnerNordström type black hole, (2.6), (2.7), which is proportional to the area (2.4), is

$$
S_{B H}=\frac{2 \pi A_{9}}{\kappa^{2}}=\frac{8 \pi^{2} L^{7}}{\kappa^{2}} \sqrt{P_{1} P_{2} P_{3} Q}=2 \pi \sqrt{n_{1} n_{2} n_{3} N} .
$$

This agrees with the expression found directly in $D=4$ [2, 3, 12, 13].

In the case of the $2 \perp 2 \perp 5 \perp 5$ configuration we find (for each pair of 2-brane and 5-brane charges) $q_{2}=\frac{4 \pi L^{5}}{\sqrt{2} \kappa} Q, q_{5}=\frac{4 \pi L^{2}}{\sqrt{2} \kappa} P$. The Dirac condition on unit charges translates into $q_{2} q_{5}=2 \pi n_{1} n_{2}$, where $n_{1}$ and $n_{2}$ are the numbers of 2 - and 5-branes. We conclude that $Q_{1} P_{1}=\frac{\kappa^{2}}{4 \pi L^{7}} n_{1} n_{2}$. Then from (3.4) we learn that

$$
S_{B H}=\frac{2 \pi A_{9}}{\kappa^{2}}=\frac{8 \pi^{2} L^{7}}{\kappa^{2}} \sqrt{Q_{1} P_{1} Q_{2} P_{2}}=2 \pi \sqrt{n_{1} n_{2} n_{3} n_{4}}
$$

9 In [30] it was argued that the 2-brane tension, $T_{2}$, satisfies $\kappa^{2} T_{2}^{3}=\pi^{2} / m_{0}$, where $m_{0}$ is a rational number that was left undetermined. The argument of [28], as well as our procedure [9], unambiguously fix $m_{0}=1 / 2$. 
Remarkably, this result does not depend on the particular choice of M-brane quantization condition (choice of $m_{0}=\pi^{2} \kappa^{-2} T_{2}^{-3}$ ) or use of D-brane tension expression since the $2 \perp 2 \perp 5 \perp 5$ configuration contains equal number of 2 -branes and 5 -branes. This provides a consistency check. Note also that the $D=4$ black holes obtained from the $2 \perp 2 \perp 5 \perp 5$ and from the $5 \perp 5 \perp 5$ M-theory configurations are not identical, but are related by U-duality. The equality of their entropies provides a check of the U-duality.

The same expression is obtained for the entropy of the $D=10$ configuration $3 \perp 3 \perp 3 \perp 3$ (4.3) (or related $D=4$ black hole). Each 3-brane charge $q_{3}$ is proportional to the corresponding coefficient $Q$ in the harmonic function (cf. (5.2))

$$
q_{3}=\frac{1}{\sqrt{2}}\left(\frac{\omega_{d+1} d}{\sqrt{2} \kappa_{10}}\right) Q \rightarrow \frac{\omega_{2} L^{3}}{2 \kappa_{10}} Q=\frac{2 \pi L^{3}}{\kappa_{10}} Q
$$

where $\kappa_{10}^{2} / 8 \pi$ is the 10 -dimensional Newton's constant and the overall factor $\frac{1}{\sqrt{2}}$ is due to the dyonic nature of the 3-brane. The charge quantization in the self-dual case implies (see [9] $q_{3}=n \sqrt{\pi}$ (the absence of standard $\sqrt{2}$ factor here effectively compensates for the 'dyonic' $\frac{1}{\sqrt{2}}$ factor in the expression for the charge). 10 Thus, $Q_{i}=\frac{\kappa_{10}}{2 \sqrt{\pi}} n_{i}$, and the area (4.4) leads to the following entropy,

$$
S_{B H}=\frac{2 \pi A_{8}}{\kappa_{10}^{2}}=\frac{8 \pi^{2} L^{6}}{\kappa^{2}} \sqrt{Q_{1} Q_{2} Q_{3} Q_{4}}=2 \pi \sqrt{n_{1} n_{2} n_{3} n_{4}} .
$$

\subsection{Counting of the microscopic states}

The presence of the factor $\sqrt{N}$ in $S_{B H}$ (5.11) immediately suggests an interpretation in terms of the massless states on the string common to all three 5-branes. Indeed, it is well-known that, for a $1+1$ dimensional field theory with a central charge $c$, the entropy of left-moving states with momentum $2 \pi N / L$ is, for sufficiently large $N$, given by 11

$$
S_{\text {stat }}=2 \pi \sqrt{\frac{1}{6} c N}
$$

We should find, therefore, that the central charge on the intersection string is, in the limit of large charges, equal to

$$
c=6 n_{1} n_{2} n_{3} .
$$

10 This agrees with the D3-brane tension, $\kappa_{10} T_{3}=\sqrt{\pi}$, since in the self-dual case $q_{p}=\kappa_{10} T_{p}$.

11 As pointed out in [31], this expression is reliable only if $N \gg c$. Requiring $N$ to be much greater than $n_{1} n_{2} n_{3}$ is a highly asymmetric choice of charges. If, however, all charges are comparable and large, the entropy is dominated by the multiply wound 5-branes, which we discuss at the end of this section. 
The fact that the central charge grows as $n_{1} n_{2} n_{3}$ suggests the following picture. 2-branes can end on 5-branes, so that the boundary looks like a closed string [20, 32, 33]. It is tempting to associate the massless states with those of 2-branes attached to 5-branes near the intersection point. Geometrically, we may have a two-brane with three holes, each of the holes attached to different 5-dimensional hyperplanes in which the 5 -branes lie. Thus, for any three 5-branes that intersect along a line, we have a collapsed 2-brane that gives massless states in the $1+1$ dimensional theory describing the intersection. What is the central charge of these massless states? From the point of view of one of the 5-branes, the intersection is a long string in $5+1$ dimensions. Such a string has 4 bosonic massless modes corresponding to the transverse oscillations, and 4 fermionic superpartners. Thus, we believe that the central charge arising from the collapsed 2-brane with three boundaries is $4\left(1+\frac{1}{2}\right)=6.12$

The upshot of this argument is that each triple intersection contributes 6 to the central charge. Since there are $n_{1} n_{2} n_{3}$ triple intersections, we find the total central charge $6 n_{1} n_{2} n_{3}$. One may ask why there are no terms of order $n_{1}^{3}$, etc. This can be explained by the fact that all parallel 5-branes are displaced relative to each other, so that the 2-branes produce massless states only near the intersection points.

One notable feature of our argument is that the central charge grows as a product of three charges, while in all D-brane examples one found only a product of two charges. We believe that this is related to the peculiar $n^{3}$ growth of the near-extremal entropy of $n$ coincident 5-branes found in [9] (for coincident D-branes the near-extremal entropy grows only as $n^{2}$ ). This is because the intersecting D-brane entropy comes from strings which can only connect objects pairwise. The 2-branes, however, can connect three different 5 -branes. Based on our observations about entropy, we conjecture that the geometries where a 2-brane connects four or more 5-branes are forbidden (otherwise, for instance, the near-extremal entropy of $n$ parallel 5-branes would grow faster than $n^{3}$ ). Perhaps such configurations are not supersymmetric and do not give rise to massless states.

The counting argument presented above applies to the configuration where there are $n_{1}$ parallel 5-branes in the (14567) hyperplane, $n_{2}$ parallel 5 -branes in the (12367) hyperplane, and $n_{3}$ parallel 5 -branes in the (12345) hyperplane. As explained in [31], if $n_{1} \sim n_{2} \sim$ $n_{3} \sim N$ we need to examine a different configuration where one replaces a number of disconnected branes by a single multiply wound brane. Let us consider, therefore, a single 5 -brane in the (14567) hyperplane wound $n_{1}$ times around the $y_{1}$-circle, a single 5-brane in the (12367) hyperplane wound $n_{2}$ times around the $y_{1}$-circle, and a single 5-brane in

12 Upon compactification on $T^{7}$, these massless modes are simply the small fluctuations of the long string in $4+1$ dimensions which is described by the classical solution (2.5). One should be able to confirm that the central charge on this string is equal to 6 by studying its low-energy modes. 
the (12345) hyperplane wound $n_{3}$ times around the $y_{1}$-circle. Following the logic of [31], one can show that the intersection string effectively has winding number $n_{1} n_{2} n_{3}$ : this is because the 2-brane which connects the three 5 -branes needs to be transported $n_{1} n_{2} n_{3}$ times around the $y_{1}$-circle to come back to its original state.13 Therefore, the massless fields produced by the 2 -brane effectively live on a circle of length $n_{1} n_{2} n_{3} L$. This implies [34 that the energy levels of the $1+1$ dimensional field theory are quantized in units of $2 \pi /\left(n_{1} n_{2} n_{3} L\right)$. In this theory there is only one species of the 2-brane connecting the three 5 -branes; therefore, the central charge on the string is $c=6$. The calculation of BPS entropy for a state with momentum $2 \pi N / L$, as in [34,31], once again reproduces (5.11). While the end result has the form identical to that found for the disconnected 5-branes, the connected configuration is dominant when all four charges are of comparable magnitude [31]. Now the central charge is fixed, and the large entropy is due to the growing density of energy levels.

\section{Black Hole Entropy in $D=5$ and Discussion.}

The counting arguments presented here are plausible, but clearly need to be put on a more solid footing. Indeed, it is not yet completely clear what rules apply to the 11dimensional M-theory (although progress has been made in [14]). The rule associating massless states to collapsed 2-branes with three boundaries looks natural, and seems to reproduce the Bekenstein-Hawking entropy of extremal black holes in $D=4$. Note also that a similar rule can be successfully applied to the case of the finite entropy $D=5$ extremal dyonic black holes described in 11 dimensions by the 'boosted' $2 \perp 5$ configuration [15]. Another possible $D=11$ embedding of the $D=5$ black hole is provided by $2 \perp 2 \perp 2$ configuration [15]. The relevant $D=10$ type IIB configuration is $3 \perp 3$ (cf. (4.3)) with momentum flow along common string. In the case of $2 \perp 5$ configuration the massless degrees of freedom on the intersection string may be attributed to a collapsed 2-brane with a hole attached to the 5-brane and one point attached to the 2-brane. If the 5-brane is wound $n_{1}$ times and the 2-brane $-n_{2}$ times, the intersection is described by a $c=6$

13 The role of $n_{1} n_{2} n_{3}$ as the effective winding number is suggested also by comparison of the $D=5$ solitonic string metric, (2.5), with the fundamental string metric, $d s^{2}=V^{-1}(d u d v+$ $\left.K d u^{2}\right)+d x_{s} d x_{s}$, where the coefficient in the harmonic function $V$ is proportional to the tension times the winding number of the source string (see e.g. [27]). After a conformal rescaling, (2.5) takes the fundamental string form with $V=H^{3}=\left(F_{1} F_{2} F_{3}\right)^{-1}$ so that near $r=0$ the $d u d v$ part of it is multiplied by $P_{1} P_{2} P_{3} \sim n_{1} n_{2} n_{3}$. Thus, the source string may be thought of as wound $n_{1} n_{2} n_{3}$ times around the circle. 
theory on a circle of length $n_{1} n_{2} L$. Following the arguments of [31, we find that the entropy of a state with momentum $2 \pi N / L$ along the intersection string is

$$
S_{\text {stat }}=2 \pi \sqrt{n_{1} n_{2} N} \text {. }
$$

This seems to supply a microscopic M-theory basis, somewhat different from that in [14], for the Bekenstein-Hawking entropy of $D=5$ extremal dyonic black holes.

We would now like to show that (6.1) is indeed equal to the expression for the Bekenstein-Hawking entropy for the 'boosted' $2 \perp 5$ configuration [15] (cf. (5.11) )

$$
S_{B H}=\frac{2 \pi A_{9}}{\kappa^{2}}=\frac{4 \pi^{3} L^{6}}{\kappa^{2}} \sqrt{Q P Q^{\prime}} .
$$

$Q$ and $P$ are the parameters in the harmonic functions corresponding to the 2-brane and the 5-brane, and $Q^{\prime}$ is the parameter in the 'boost' function, i.e. $T^{-1}=1+Q / r^{2}$, $F^{-1}=1+P / r^{2}, K=1+Q^{\prime} / r^{2}$. Note that here (cf. (5.1])

$$
Q^{\prime}=\frac{\kappa^{2} N}{\pi L^{7}}, \quad q_{2}=\frac{4 \pi^{2} L^{4}}{\sqrt{2} \kappa} Q, \quad q_{5}=\frac{4 \pi^{2} L}{\sqrt{2} \kappa} P .
$$

As in the case of the $2 \perp 2 \perp 5 \perp 5$ configuration, we can use the Dirac quantization condition,

$q_{2} q_{5}=2 \pi n_{1} n_{2}$, to conclude that $Q P=\frac{\kappa^{2}}{4 \pi^{3} L^{5}} n_{1} n_{2}$. This yields (6.1) when substituted into (6.2). A similar expression for the BPS entropy is found in the case of the completely symmetric $2 \perp 2 \perp 2$ configuration,

$$
S_{B H}=\frac{4 \pi^{3} L^{6}}{\kappa^{2}} \sqrt{Q_{1} Q_{2} Q_{3}}=2 \pi \sqrt{n_{1} n_{2} n_{3}},
$$

where we have used the 2-brane charge quantization condition (5.8), which implies that $Q_{i}=n_{i} L^{-4}\left(\frac{\kappa}{\sqrt{2} \pi}\right)^{4 / 3}$. Agreement of different expressions for the $D=5$ black hole entropy provides another check on the consistency of (5.8),(5.9).

Our arguments for counting the microscopic states applies only to the configurations where M-branes intersect over a string. It would be very interesting to see how approach analogous to the above might work when this is not the case. Indeed, black holes with finite horizon area in $D=4$ may also be obtained from the $2 \perp 2 \perp 5 \perp 5$ configuration in Mtheory, and the $3 \perp 3 \perp 3 \perp 3$ one in type IIB, while in $D=5$ - from the $2 \perp 2 \perp 2$ configuration. Although from the $D=4,5$ dimensional point of view these cases are related by U-duality to the ones we considered, the counting of their states seems to be harder at the present level of understanding. We hope that a more general approach to the entropy problem, which covers all the solutions we discussed, can be found.

\section{Acknowledgements}

We are grateful to V. Balasubramanian, C. Callan and M. Cvetič for useful discussions. I.R.K. was supported in part by DOE grant DE-FG02-91ER40671, the NSF Presidential Young Investigator Award PHY-9157482, and the James S. McDonnell Foundation grant No. 91-48. A.A.T. would like to acknowledge the support of PPARC, ECC grant SC1*CT92-0789 and NATO grant CRG 940870. 


\section{References}

[1] A. Sen, Mod. Phys. Lett. A10 (1995) 2081, hep-th/9504147.

[2] F. Larsen and F. Wilczek, hep-th/9511064.

[3] M. Cvetič and A.A. Tseytlin, Phys. Rev. D53 (1996) 5619, hep-th/9512031.

[4] A. Strominger and C. Vafa, hep-th/9601029.

[5] R. Kallosh, A. Linde, T. Ortín, A. Peet and A. van Proeyen, Phys. Rev. D46 (1992) 5278.

[6] M. Cvetič and D. Youm, Phys. Rev. D53 (1996) 584, hep-th/9507090.

[7] M. Cvetič and A.A. Tseytlin, Phys. Lett. B366 (1996) 95, hep-th/9510097.

[8] A.A. Tseytlin, Mod. Phys. Lett. A11 (1996) 689, hep-th/9601177.

[9] I.R. Klebanov and A.A. Tseytlin, hep-th/9604089 (revised).

[10] M. Cvetič and D. Youm, unpublished.

[11] C.G. Callan and J.M. Maldacena, hep-th/9602043.

[12] J.M. Maldacena and A. Strominger, hep-th/9603060.

[13] C. Johnson, R. Khuri and R. Myers, hep-th/9603061.

[14] R. Dijkgraaf, E. Verlinde and H. Verlinde, hep-th/9603126; hep-th/9604055.

[15] A.A. Tseytlin, hep-th/9604035.

[16] V. Balasubramanian and F. Larsen, hep-th/9604189.

[17] M.B. Green and M. Gutperle, hep-th/9604091.

[18] M. Cvetič and A. Sen, unpublished.

[19] G. Papadopoulos and P.K. Townsend, hep-th/9603087.

[20] A. Strominger, hep-th/9512059.

[21] G. Papadopoulos, hep-th/9604068.

[22] R. Güven, Phys. Lett. B276 (1992) 49.

[23] G.T. Horowitz and A. Strominger, Nucl. Phys. B360 (1991) 197.

[24] M.J. Duff and K.S. Stelle, Phys. Lett. B253 (1991) 113.

[25] M.J. Duff and J.X. Lu, Phys. Lett. B273 (1991) 409.

[26] G.W. Gibbons and K. Maeda, Nucl. Phys. B298 (1988) 741.

[27] A. Dabholkar, J.P. Gauntlett, J.A. Harvey and D. Waldram, hep-th/9511053.

[28] J.H. Schwarz, hep-th/9510086.

[29] J. Polchinski, S. Chaudhuri and C.V. Johnson, hep-th/9602052.

[30] M.J. Duff, J.T. Liu and R. Minasian, Nucl. Phys. B452 (1995) 261, hep-th/9506126.

[31] J. Maldacena and L. Susskind, hep-th/9604042.

[32] P.K. Townsend, hep-th/9512062.

[33] K. Becker and M. Becker, hep-th/9602071.

[34] S. Das and S. Mathur, hep-th/9601152. 